\title{
Molecular therapy for duchenne muscular dystrophy (DMD) when should treatment begin?
}

\author{
Byron A. Kakulas* \\ Perron Institute for Neurological and Translational Research, Perth Western, Australia
}

\section{Letter to Editor}

The discovery and development of antisense oligonucleotide (AO) treatment for DMD based on alternate splicing and exon skipping has opened a new era of treatment for genetic diseases [1]. In September 2016 the FDA approved AO as a treatment for DMD and it is now commercially available under the trade names Eteplirsen, Exondys 51 (Sarepta Therapeutics). Morpholino delivered AOs have proven effective in delaying the deterioration in muscle function in DMD [1]. This great advance calls for a review of the pathology and natural history of DMD as a guide to the optimal time for treatment to begin.

High levels of serum creatine phosphate at birth and morphological changes in skeletal muscle in the foetus shows that DMD begins in utero even though the clinical diagnosis is usually established when the boy is about 4 or 5 years of age. Muscle biopsy of the gastrocnemius muscle at this time reveals flagrant disease with polyfocal necrosis, abortive regeneration, loss of muscle fibres and excessive fibrosis [2]. Independent ambulation usually ceases at the ages of 12 or 13 years and the boy becomes wheelchair bound. At this stage the large limb muscles such as the quadriceps femoris are at the "end stage" with almost total loss of muscle fibres which are replaced by fat and fibrous tissue [3].

The gene for DMD on the $\mathrm{X}$ chromosome produces a very large cytoskeletal protein aptly termed dystrophin which underlies and gives strength to the muscle membrane. As one of the largest genes in the human genome dystrophin is prone to mutations usually in the form of DNA deletions. In DMD dystrophin is totally absent so that the membrane disrupts under normal contraction. The disruption allows the entry of calcium which then activates destructive enzymes. The pathogenesis of DMD was first elucidated in an animal model, the Rottnest Island Quokka, a small Australian marsupial which is subject to muscle paralysis when kept in small cages. The disorder was proved to be due to antioxidant deficiency and when corrected by the administration of Vitamin E miraculously the affected muscles completely regenerated [4].

This great discovery of total muscle regeneration proved that all skeletal muscle disorders are potentially curable when the cause of its breakdown is remedied [4]. Thus, DMD is potentially a curable disease but the time to commence exon skipping /alternate splicing AO treatment is critical in the light of its natural history. Because DMD is a continuously progressive disorder it is evident that the sooner the treatment is commenced the better the outcome will be. Alternatively, very little benefit will result if treatment is given at the "end stage" of the disease process. The momentous discovery of the potential for muscle to completely regenerate according to the pioneer work in the Rottnest Quokka should be taken to its best advantage.

\section{References}

1. Le BT, Veedu RN, Fletcher S, Wilton SD (2017) Antisense Oligonucleotide Development for the Treatment of Muscular Dystrophies. Expert Opinion Orph Drugs 4 (2): 139-152.

2. Mastaglia FL, Kakulas BA (1969) Regeneration in Duchenne muscular dystrophy. A histological and histochemical study. Brain 92: 809-818.

3. Kakulas BA, Adams RD (1985) Diseases of muscle: Pathological foundations of clinical myology. 4th Edn, Harper and Row Publishers, Philadelphia, 1985.

4. Kakulas BA (1961) Myopathy affecting the Rottnest quokka (Setonix brachyurus) reversed by $\alpha$ tocopherol. Nature 191: 402-403. [Crossref]
Copyright: (C2018 Kakulas BA. This is an open-access article distributed under the terms of the Creative Commons Attribution License, which permits unrestricted use, distribution, and reproduction in any medium, provided the original author and source are credited.
Correspondence to: Byron A. Kakulas, Perron Institute for Neurological and Translational Research, Australia, E-mail: byron.kakulas@perron.uwa.edu.au

Received: March 08, 2018; Accepted: March 18, 2018; Published: March 20, 2018 